\title{
Invariant slow manifolds of an Atomic Force Microscope system under the Derjaguin-Muller-Toporov forces and a slow harmonic base motion
}

\author{
Sara Baghdadi, Faouzi Lakrad and Mohamed Belhaq \\ Laboratory of Renewable Energy and Dynamics of Systems, Faculty of Sciences Ain Chock, \\ University Hassan II-Casablanca, Casablanca, Morocco
}

\begin{abstract}
In the present work, we study the nonlinear vibrations of an AFM system, modeled as a linear massspring-damper system, under the Derjaguin-Muller-Toporov forces and subject to imposed slow harmonic base displacement. The invariant slow manifolds of the system are approximated and their bifurcations are investigated. Then, the charts of behaviors of the different operating modes of the AFM are determined. The dynamic saddle-node bifurcations of the contact and the noncontact invariant slow manifolds are found to be responsible for the occurrence of the tapping mode.
\end{abstract}

\section{Introduction}

Atomic Force Microscopes (AFM) are employed in a broad spectrum of applications such as imaging and characterization with an atomic resolution and in chemical and biological analysis, see for instance [1] and [2].

Atomic force microscopy is based mainly on a vibrating microcantilever with a nano-scale tip that interacts with a sample surface via short-and long-range intermolecular forces. An enhanced understanding of these vibrations is central to the correct interpretation of the AFM outputs.

Lakrad [3] and Khadraoui et al. [4] studied the nonlinear dynamics of the AFM under the Lennard-Jones force between the tip and the sample. The micro-cantilever was subject to an imposed slow harmonic displacement of its base. They computed the slow manifolds in order to explain the occurrence of the contact, noncontact and tapping modes of the AFM.

The present paper follows the same workflow than [3] but in the presence of the Derjaguin-Muller-Toporov (DMT) interaction forces between the tip and the sample. The AFM is modelled by a single degree-of-freedom lumped linear system subject to a slow harmonic base displacement. The nonlinearities are introduced in the system through tip-sample interaction forces.

The present work is organized as follows: in Section 2 the mathematical model of the AFM is presented. Section 3 contains the results of static and linear free vibration analysis. In Section 4, the theory of slow manifolds is used to determine various operating modes of the AFM.

\section{Mathematical modeling}

A single degree-of-freedom model, depicted in Fig.1, is used to represent a lumped-parameters model of an Atomic Force Microscope. The microcantilver is modeled as a sphere of radius $R$ and mass $m$, its equivalent spring rigidity and viscous damping coefficient are denoted $K$ and $c$, respectively. Moreover, the base of the microcantilver is excited by a piezoelectric actuator generating a vertical harmonic displacement $V(t)=v^{*} \cos (\Omega t)$.

Furthermore, the cantilever-tip-sample interaction is modeled by the van der Waals force in the non-contact phase and by the Derjaguin Muller-Toporov (DMT) forces in the contact phase. The DMT model is valid for describing stiff contacts with adhesion forces and small tip radii [5].

The equation governing the absolute displacement $x(t)$ of

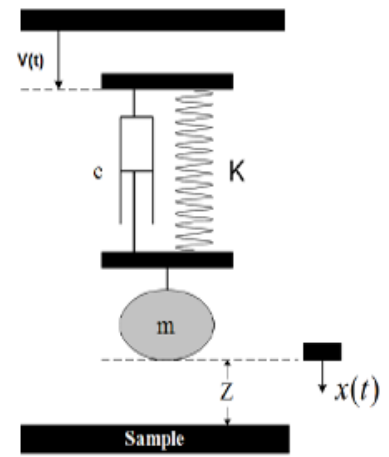

Figure 1. The studied lumped-parameters model of the AFM.

the mass towards the sample is given by

$$
m \ddot{x}(t)+c(\dot{x}(t)-\dot{V}(t))+K(x(t)-V(t))=F
$$

where a dot indicates a derivative with respect to time $t, Z$ is the initial gap between the AFM tip and the sample, and $F$ is the interaction force between the mass and the sample. This latter depends piecewisely on the instantaneous gap, it is expressed as follows

$$
F= \begin{cases}F_{v d W}, & \text { for } Z-x(t) \geq a_{0} \\ F_{D M T}, & \text { for } Z-x(t)<a_{0}\end{cases}
$$

where $F_{v d W}$ is the non-contact attractive force of van der Waals and $F_{D M T}$ represents the Derjaguin-Muller-Toporov 
contact force. The DMT force is composed of the long range attractive van der Waals and the short-range repulsive Hertzian contact forces [6]. These forces are given by

$$
\begin{aligned}
F_{v d W} & =\frac{H R}{6(Z-x(t))^{2}} \\
F_{D M T} & =\frac{H R}{6 a_{0}^{2}}-\frac{4}{3} E^{*} \sqrt{R}\left[a_{0}-(Z-x(t))\right]^{\frac{3}{2}}
\end{aligned}
$$

where $a_{0}$ represents the intermolecular distance at which the contact is initiated; $H, R$, and $E^{*}$ are, respectively, the Hamaker contant, the tip radius, and the effective modulus of elasticity. The latter is given by

$$
\frac{1}{E^{*}}=\frac{1-v_{m}^{2}}{E_{m}}+\frac{1-v_{s}^{2}}{E_{s}}
$$

with $v_{m}$ and $E_{m}$ are, respectively, the Poisson ratio and modulus of elasticity of the micro-cantilever and similarly $v_{s}$ and $E_{s}$ are those of the sample.

The equation of motion (1), written in nondimensional form, can be expressed as follows

$$
y^{\prime \prime}(\tau)+2 \xi y^{\prime}(\tau)+y(\tau)=\left(1-\frac{V(\tau)}{Z}\right)-2 \xi \frac{V^{\prime}(\tau)}{Z}-\tilde{F}
$$

where distances were normalized by the initial gap $Z$.

- $\tau$ is a new scale of time : $\tau=\omega t$, with $\omega=\sqrt{K / m}$ is the linear natural frequency of the micro-cantilever in the absence of the sample interaction forces. The prime is the derivative with respect to $\tau$.

$-\xi$ is the damping ratio: $\xi=c /(2 m \omega)$.

$-y$ is the nondimensional instantaneous tip/sample gap: $y=(Z-x) / Z$.

- The nondimensional imposed displacement $V(\tau) / Z=$ $f \cos (\epsilon \tau)$, where $f=v^{*} / Z$ and $\epsilon=\Omega / \omega$.

The tip/sample interaction force can be rewritten

$$
\tilde{F}=\left\{\begin{array}{lll}
\frac{\gamma_{2}}{y(\tau)^{2}} & \text { for } & y(\tau) \geq A_{0} \\
\frac{\gamma_{2}}{A_{0}^{2}}-\gamma_{1}\left[A_{0}-y(\tau)\right]^{\frac{3}{2}} & \text { for } & y(\tau)<A_{0}
\end{array}\right.
$$

where $\gamma_{1}=\frac{4}{3} \frac{E^{*} \sqrt{R Z}}{K} ; \gamma_{2}=\frac{H R}{6 K Z^{3}}$ and $A_{0}=\frac{a_{0}}{Z}$.

\section{Static equilibria and free vibrations}

In the present section we will compte the static equilibria of Eq.(6) and the natural frequencies of the free vibrations around them.

\subsection{Static equilibria}

The static study of the model is obtained by dropping the time derivatives and cancelling the base motion in Eq.(6). Then, the static equilibria $y_{s}$ are obtained by solving the following third order algebraic equations

$$
\left\{\begin{array}{lll}
y_{s}^{3}-y_{s}^{2}+\gamma_{2}=0 & \text { for } & y_{s} \geq A_{0} \\
y_{s}=1-\frac{\gamma_{2}}{A_{0}^{2}}+\gamma_{1}\left(A_{0}-y_{s}\right)^{\frac{3}{2}} & \text { for } & y_{s}<A_{0}
\end{array}\right.
$$

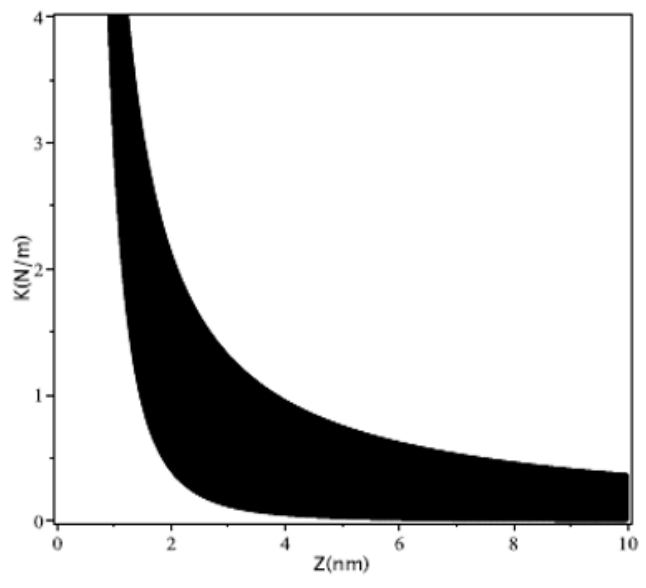

Figure 2. Number of static equilibria of Eqs. (8) in the $(Z, K)$ plane. The black zone corresponds to the existence of three equilibria, and the white zone to one equilibrium.

Figure 2 shows the number of static equilibria in the plane of the stiffness $K$ of the system versus the initial gap $Z$. The black zone corresponds to the existence of three static equilibria and the white zone to the existence of one static equilibrium. In fact, the contact zone i.e., $y_{s}<A_{0}$ has only one static equilibrium, while the non-contact zone $y_{s} \geq A_{0}$ can have three solutions for $\gamma_{2}<\frac{4}{27}$.

It is worth noting that the system can have one static equilibrium for hard micro-cantilevers and for high initial gaps. Figure 3 shows, for $K=0.89 \mathrm{~N} / \mathrm{m}$, the static mass-sample

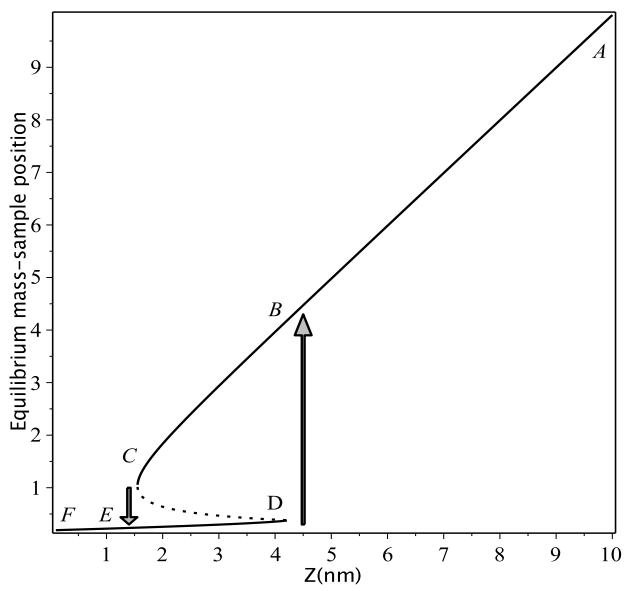

Figure 3. Static tip/sample separation versus $Z$.

separation distance versus the initial gap $Z$. The resulting static curve can be divided into two regions; a bistable region, for $Z \in[1.5 \mathrm{~nm}, 4.2 \mathrm{~nm}]$, formed by the stable branches $\mathrm{BC}, \mathrm{ED}$ and the unstable branch $\mathrm{CD}$ and a monostable region formed by the stable branches $\mathrm{AB}$ and $\mathrm{EF}$.

The bistable region is delimited by two saddle-node bifurcations at points $C$ and $D$ where jumps occur when varying the initial gap $Z$.

\subsection{Free vibrations}

In order to compute the natural frequencies of the system under the effects of the interaction forces, the nondimen- 
sional Eqs.(6) and (7) are linearized around a given stable static equilibrium $y_{s}$. Moreover, the damping and the imposed base motion are cancelled.

The normalized natural frequencies of the contact mode $f_{r e q}$ and the noncontact mode $f r e q_{n c}$ are given by

$$
\begin{aligned}
& \text { freq }_{c}=\frac{1}{2 \pi} \sqrt{1+\frac{3 \gamma_{1}}{2} \sqrt{A_{0}}-y_{s}} \text { for } y_{s}<A_{0} \\
& \text { freq }_{n c}=\frac{1}{2 \pi} \sqrt{1-2 \frac{\gamma_{2}}{y_{s}^{3}}} \quad \text { for } \quad y_{s} \geq A_{0}
\end{aligned}
$$

In the absence of the interaction forces, the contact and noncontact frequencies are constant and equal to $1 /(2 \pi)$. In Fig. 4 are shown, versus the initial gap $Z$, numerical and

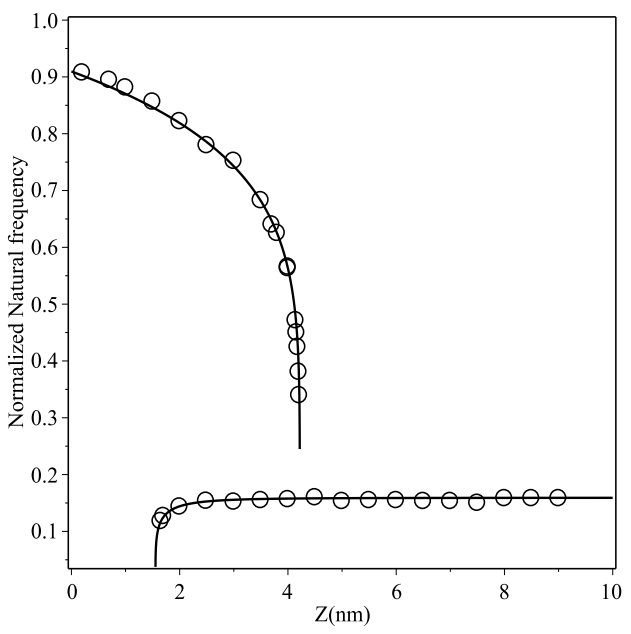

Figure 4. Normalized natural frequency versus the initial gap $Z$. Circles are obtained numerically from Eq.(6). Continuous lines are analytically computed frequencies in Eqs. (9) and (10).

analytical normalized natural frequencies given by equations (9) and (10), respectively, for contact and noncontact modes. The numerically computed frequencies are obtained by integrating Eq.(6) using a fourth order RungeKutta algorithm, then the corresponding power spectrum is computed using a Fast Fourier Transformation algorithm. Figure 4 shows that in the contact phase, the natural frequency (9) is decreasing with increasing the initial gap $Z$. This can be explained by the decrease of the apparent stiffness of the micro-cantilever. In the non-contact phase, increasing the initial gap $Z$ leads to the increase of the natural frequency (10) till reaching the natural frequency of the system without any force of interaction i.e., $1 / 2 \pi$. In short we have two zones: one with one solution corresponding to monostable region, and the other zone with two stable solution that represents the bistable region.

\section{Dynamic behavior}

In the present paper, the frequency of the base displacement is taken very small with respect to the natural frequency of the system i.e., $\epsilon=\Omega / \omega<<1$. The case of resonant excitations was investigated in many papers, see for instance [7].

Equation (6) can be written as a fast-slow system since it is ruled by two dynamics: the first on the time scale of order 1 (the natural frequency) and the second one on the time scale of order $\epsilon$ (the base displacement).

Introducing the new slow time scale $T=\epsilon \tau$, Eq.(6) becomes

$$
\begin{aligned}
\epsilon^{2} \frac{d^{2} y}{d T}+2 \epsilon \xi \frac{d y}{d T}+y & =1-f \cos (T) \\
& +2 \epsilon \xi f \sin (T)-\tilde{F}(T)
\end{aligned}
$$

In the limit $\epsilon \rightarrow 0$ one can compute an approximation of the slow manifolds by solving the following algebraic equations

$$
\begin{aligned}
& y^{3}+(f \cos (T)-1) y^{2}+\gamma_{2}^{2}=0, \quad \text { for } \quad y \geq A_{0} \\
& y-\gamma_{1}\left(A_{0}-y\right)^{\frac{3}{2}}+f \cos (T)=1-\frac{\gamma_{2}}{A_{0}^{2}}, \text { for } y_{s}<A_{0}
\end{aligned}
$$

The invariant slow manifolds can be viewed as periodic quasi-static lines of equilibria with respect to the slow time scale $T$. During a period of $T$ they can bifurcate giving rise to very interesting phenomena [8]. Indeed, the computation of the invariant slow manifolds and their bifurcations are of great importance for determining and understanding the dynamics of the AFM system ruled by Eq.(1). In fact, solutions of the system will spend most of their time following attracting slow manifolds. This phenomenon is sometimes called "slaving principle" or adiabatic reduction [9].

Figure 5 shows the graphs of the slow manifolds versus the slow time T, for various values of the amplitude $f$ of the imposed harmonic displacement of the base and for an initial gap $Z=3 \mathrm{~nm}$, belonging to the bistable static region, see Fig. 4. The stable manifolds are plotted in continuous lines and unstable ones with dashed lines. The lower and the upper stable solutions correspond to contact and noncontact slow manifolds, respectively.

In Fig. 5a, for $f=0$ i.e., no base motion, the slow manifolds are the coexisting three static equilibria, that are independent of $T$, corresponding to the contact and noncontact modes and separated by an unstable solution. When increasing $f$ the slow manifolds are becoming periodic with increasing amplitudes. Thus, for $f=0.39$ the contact slow manifold collides with the unstable manifold leading to a sadlle-node bifurcation. Consequently, for $f=0.45$ the contact slow manifold does not exist for all times and the noncontact slow manifold is the visited solution. Increasing further $f$ leads to a new collision between the noncontact slow manifold and the unstable slow manifold. Hence, for $f=0.55$, the contact and the non contact slow manifolds will be both visited during a period of the base displacement. This solution is geometrically a periodic burster and physically corresponds to a tapping mode of the AFM [3]. The confirmation that the solutions of the nondimensional Eq.(6) are following the scenario discussed previously is shown in Fig. 6 for $Z=3 \mathrm{~nm}$.

Based on the computed slow manifolds, Fig. 7 shows the behavior chart of the system in the plane $(f, Z)$. In the white region there exist only one solution: either the contact solution in the lower zone or the nonconact solution in the upper zone. In the light grey zone, the two solutions coexist, but only one is chosen depending on the initial conditions. In the dark grey zone, the two solutions will be visited during a period of the base motion: the tapping mode. 


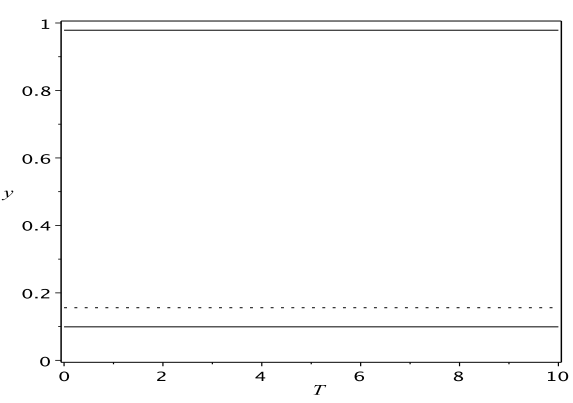

(a) $f=0$

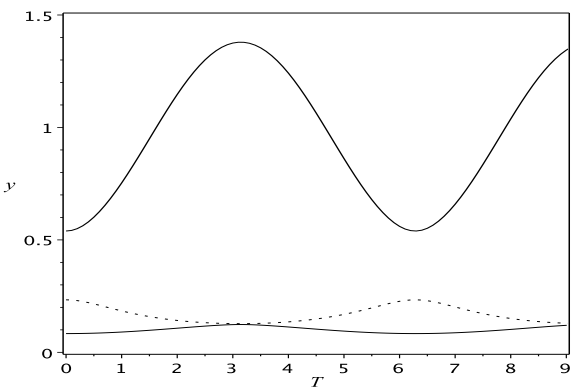

(b) $f=0.39$

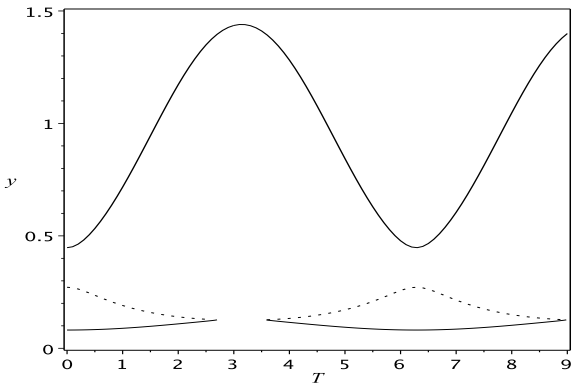

(c) $f=0.45$

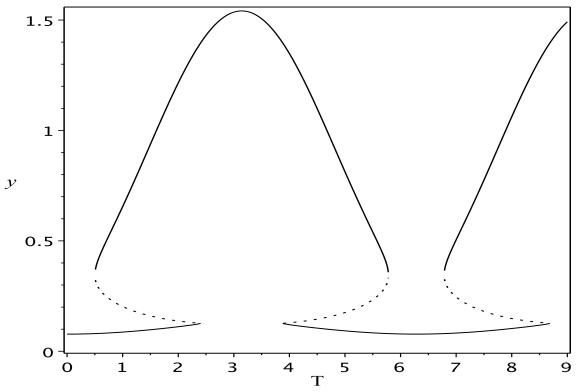

(d) $f=0.55$

Figure 5. Graphs of the slow manifolds, given by Eqs.(12) and (13), for various base amplitudes $f$ and for $Z=3 \mathrm{~nm}$

\section{Conclusion}

In the present work, we studied nonlinear dynamics of a lumped single d.o.f. model of an AFM system under the vand der waals and Derjaguin-Muller-Toporov interaction forces with very low frequency displacement of its base. The invariant slow manifolds theory is used to determine the different operating modes of the AFM (contact, noncontact and tapping modes). It is shown that the tapping mode is triggered by dynamic saddle-node bifurcations. As a continuation of the present work, the nonlinear dynamic of continuous models of the AFM will be studied under the effect of imposed slow harmonic displacements.

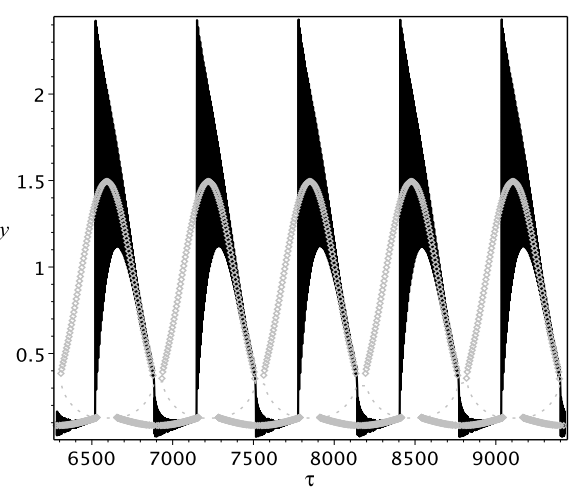

Figure 6. Numerical solutions of Eq.(6) in black and slow manifolds in grey, for $f=0.5$ and $Z=3 \mathrm{~nm}$.

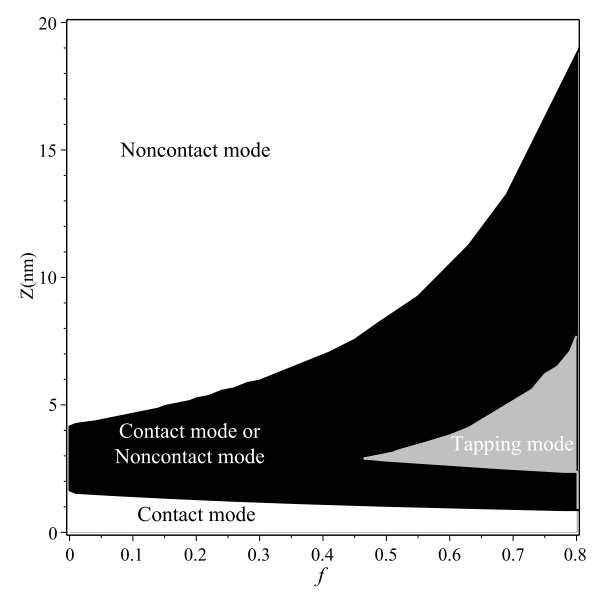

Figure 7. Chart of the system behaviors in the plane $(f, Z)$.

\section{References}

1. G. Binnig, C.F. Quate, C. Gerber. Atomic force microscope. Phys Rev Lett 56(9): 930-4, 1986.

2. P. Eaton, P. West. Atomic force microscopy. Oxford University Press, 2010.

3. F. Lakrad. Invariant slow manifolds of an Atomic Force and a slow harmonic base motion. Commun Nonlinear Sci Numer Simulat 32, 49-62, 2016.

4. M. Khadraoui , F. Lakrad, M. Belhaq. Effecrs of a slow harmonic displacement on an Atomic Force Microscope system under Lennard-Jones forces, MATEC Web of conferences 83, 04001, 2016.

5. R. Garcia. Amplitude modulation atomic force microscopy. Wiley-VCH Verlag, 2010.

6. J. Israelachvili. Intermolecular and surface forces. 2nd ed. Academic; 1991.

7. I. Manoubi, F. Najar, S. Choura, A.H. Nayfeh. Nonlinear Dynamical analysis of an AFM tapping mode microcantilever beam. MATEC Web of Conferences 1, 04002, 2012.

8. F. Lakrad, W. Schiehlen. Effects of a low frequency parametric excitation. Chaos Solitons \& Fractals 22: 1149-64, 2004.

9. N. Berglund, B. Gentz. Noise-induced phenomena in slow-fast dynamical systems. London: SpringerVerlag; 2006. 